\title{
Constella, a quick configuration platform for rapid response missions ${ }^{12}$
}

\author{
Maarten (Max) Meerman \\ Martin Sweeting \\ Surrey Satellite Technology Limited \\ And Surrey Space Centre \\ University of Surrey, Guildford, Surrey GU2 7XH \\ $+441483689278$ \\ m.meerman@sstl.co.uk
}

Abstract - Constella is a novel very quick response satellite platform that can be used in any Low Earth Orbit. It can be used as a single unit or as part of a constellation of spacecraft. It can be pre-manufactured to a large extent, and a selection of subsystems can be ready to select from, to provide last-minute configuration options for both the payload and platform. This satellite contains a number of innovations that have not been used in space before, and they will allow very quick response missions to take place. Only a very short time is required to decide on the mission and plan the satellite design, then assemble the final parts, and test and launch the satellite into orbit. The total time can be down to weeks or even days. Unlike most quick-response missions, where anything under one year is included, and where the satellite typically has to be completely ready and tested, waiting for quick call-up, the Constella can be tailored with interchangeable attitude sensors, propulsion units, communications equipment, payloads and more, just before launch, even right at the launch site if required. The platform produces almost the same amount electrical power, irrespective of orbital inclination or ascending node time, removing the need for solar panel design changes at the configuration stage.

Additionally, the design of the platform allows for optimized initial manufacture, requiring very little assembly and integration space and time, in itself a major cost saver.

The paper discusses the specific technical aspects of the design of the platform, showing the innovations that are both physical and operational. It shows proposed missions that can take advantage of this platform, allowing them to proceed where traditional rapid response or low cost engineering techniques will not be feasible.
TABLE OF CONTENTS

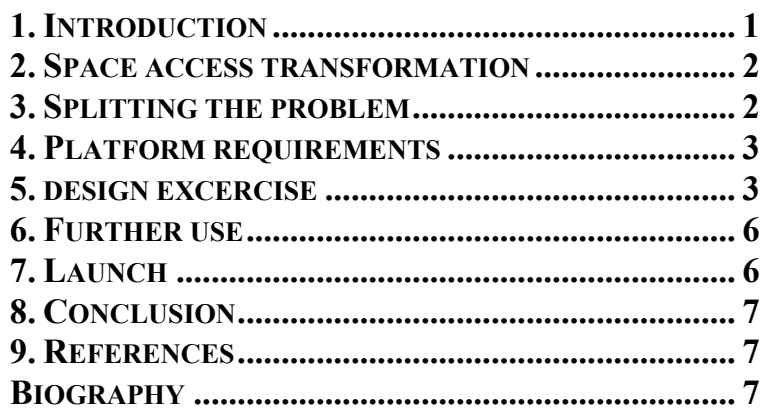

\section{INTRODUCTION}

There has been a downward trend in satellite design and manufacture over the last few years. This is a reverse from the mid space history, where after the heydays of Apollo, timescales went only up. In the early days of space development, late 1950s and early 1960s, there were no standards, and as time was the only yardstick to measure progress by, scientific and engineering development spacecraft were built and launched in quick succession. Space development ended up going to larger and costlier spacecraft, with increasingly longer timescales and higher cost. For commercial telecommunications missions, that can be planned and executed over long timescales, this is no problem. There have been developments to shorten the timescales of development of satellite missions, and every once in a while there is some proud statement announcing that a certain satellite has been designed and readied for launch in only eighteen months. It is easy, however, to imagine a mission that would be able to take advantage of a mission design-and-build period of only a few days. This approach would allow investigation of newly discovered phenomena, or would allow observation satellites to respond quickly to developing military or natural situations. Instead of having to reply on the standard LandSat type imagery, one can quickly configure an imager for the

\footnotetext{
${ }^{1} 0-7803-9546-8 / 06 / \$ 20.00 \bigodot 2006$ IEEE

${ }^{2}$ Revision 1, January 2006
} 
correct optical bands to assess the signature of a chemical spread from an industrial accident. The imager can then be launched into an orbit that is optimized for the required viewing, including local weather patterns, for instance late afternoon Sun-synchronous.

A typical spacecraft launcher integration campaign will take at least a month from shipping the satellite to the launch site to separation in orbit, and this section of the mission must be reduced as much as the satellite design, build and test time. Apart from technical issues that must be solved, the process requires buy-in from customers and launch providers.

An initial design [1] study had led to the initial development of a satellite bus, which was then further developed for a specific mission proposal that came along shortly afterwards. The outcome of the mission design was a versatile platform well suited to the mission turnaround required for quick missions. The UK TOPSat mission has since used a derivative of this platform, and the RapidEye constellation is about to use it for five spacecraft.

\section{SPACE ACCESS TRANSFORMATION}

Many mission ideas never go beyond the back of the envelope, or the end-user's desk, because the timescales involved would not allow the mission to be completed, or even started in orbit, before the subject of the mission is no longer available, or before the information would be out of date, or otherwise of no use anymore. If one could imagine a mission that could be in orbit within a day, not only could new missions be thought up, but satellites could be kept ready on the ground to quickly increase capacity or modify capability when required. This approach would obviate the need for large in-orbit capability that ties up capital and operational cost.

The space launch industry has developed from a joint missile history, but whereas missiles can be launched in an instance with little or no final preparation required, orbital launchers require lengthy procedures to be completed before every launch. With the timescales involved in typical space missions, there is no need for launches to happen quicker than at present: as long as the rocket preparation time is shorter than what is required for the satellite development there is no pressure.

There are therefore two prongs to the development of a space capability that allows true quick response: the development of a satellite that can be configured in a very short time, and the development of a quick-response launcher, either a new one or a different way of treating and processing current launchers to make them respond to opportunities faster.

\section{SPLITTING THE PROBLEM}

Space missions start as two separate problems: the spacecraft and the launch vehicle. At some point they become interlinked, through various mechanical, electrical, and other interfaces. Coupled-loads analysis is performed to ensure that the combined structure will not exhibit any undesirable vibration characteristics, and any changes on either side must be carefully controlled. The satellite will go through a fit-check exercise long before launch to ensure that there will be no integration problems at the launch site.

If a satellite can be designed with fixed mechanical parameters, it could be pre-qualified, and no coupled-loads analysis would have to take place for every mission. There would be no need for a fit-check meeting either. The launch agency could manufacture and provide the spacecraft half of the separation system, with all cables already attached, ensuring that the spacecraft integrates to the launcher without any possibility of a mismatch.

\section{Launcher standardization}

Not many launch vehicles are in current use, especially compared to the number of different spacecraft that have been launched. Changing a launch vehicle or designing a new one is a long and expensive process; therefore it is likely that it would be simpler to assess what launchers are available, and use that information as the starting point for spacecraft platform design.

\section{Satellite standardization}

To provide for a standard spacecraft, either both platform and payload must always be the same, or the platform must be able to compensate for any differences between payloads. Clearly the latter will make it simpler for payload development, but it requires ingenuity and larger margins for the platform design.

This spacecraft standardization can be compared with a typical desktop PC. Whatever the actual hardware that is selected at the point of purchase; whether a larger hard disk, more memory or a faster processor: the box will remain the same size, it will fit in the same spot that the previous PC occupied on the desk, and it will plug into the same mains outlet. There is no need for the office layout manager to take the actual specification of the computers into account when buying desks.

One of the critical design inputs here is directed optimization: look at standards rather than optimize for size. The same could apply to satellites: rather than try to shave off every last gram of mass and shrink every subsystem into the smallest volume, make the satellite standard and cheap. This will make it simpler to reuse, as unused margins may well come in handy when a satellite platform is used for the next-generation avionics. 


\section{Platform ReQuirements}

A satellite platform for standardized space access will have to be carefully designed for reconfigurability, ease of assembly and test, and ease of launcher integration.

Few, if any, of the current platforms in use result in truly standard satellites across missions. Obviously, in the case of missions like Iridium, all satellites are the same, but that is not the same as having a satellite supporting different payloads in different orbits without having to completely requalify the entire technical path from design to orbital operations.

The platform must be able to accept a ready-built payload with a minimum number of integration steps, with simple and inspectable interfaces. This means that the bolted joints and connectors have to be external in easy accessible places, even if this means a heavier or larger design. It also needs quickly reconfigurable attitude sensors and actuators, and with a thermal and power system that can cope with any orbital inclination and hour angle.

The payload accommodation can be clearly defined, so it would be easy for designers to have units ready, or very quickly design or modify a payload for the mission. The current interface specification includes a dual CAN bus for command and housekeeping telemetry, switched power at $28 \mathrm{~V}$, and LVDS serial data link. The structure includes a number of standard mounting points, each having four M4 threaded holes in a $100 \mathrm{~mm}$ square configuration. Individual sensors and antennas can either be directly compatible, or use an interface plate. The CAN-bus and the $100 \mathrm{~mm}$-square mounting were used successfully in the past on the UoSAT12 satellite, where it allowed the system qualification to progress independent from the design of individual subsystems.

\section{Pre-manufacturing}

Typical lead times for space components is many months, so the satellite must consist of pre-manufactured elements that can be assembled without requiring lengthy procedures or tests. A selection of basic satellite parts can be kept in storage ready to be assembled quickly when required. With such interchangeable parts it may be possible to define open standards to allow third-party developments to be included. The subsystems may be cheaper to make as they do not have to be individually optimized for each mission and this may offset the investment required for the manufacture of components that may not be used for a while.

\section{DESIGN EXCERCISE}

\section{Sea-surface radar mission}

That mission calls for a number of spacecraft for regular worldwide measurement of the state of the sea surface. The constellation of satellites covers the entire Earth, meaning they will be operating in different thermal and sunlight environments. When optimizing the system for Sea coverage, the best orbit turned out not to be polar, but slightly lower at 72 degrees. This produced the best coverage of shipping lanes. It is of course a non-Sunsynchronous orbit, and meaning that the Sun hour angle changes continually for each individual satellite, requiring a spacecraft design that is insensitive to the thermal and power issues related to this.

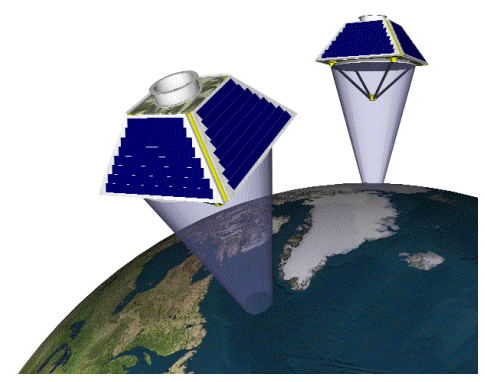

\section{Mission Requirements}

The satellite design's main requirements were to provide a 1-metre-diameter nadir pointing dish antenna, $50 \mathrm{~W}$ of orbit-average power, and all Sun angle power generation. The satellite needs some on-board propulsion to acquire and maintain position in the constellation. Each spacecraft should also be cheap to build and launch, as the constellation would consist of 16 satellites, and it was planned for a commercial business need, which placed strict financial limits on the program.

Cost and volume restrictions meant that there was no option of providing full redundancy for every subsystem; however, the constellation will have to keep working for a seven-year period minimum to meet the business plan. The propellant usage, battery life, radiation degradation of solar panels and other predictable lifetime effects can be taken into account by suitable sizing of the designs, but random failures are more difficult to cope with. This reliability required some creative strategies to be developed for dealing with various failure scenarios.

\section{Satellite design}

Initially the satellite was to be based on an existing platform, as that was deemed the cheapest solution, apparently requiring little design work. The selected platform was a $100-\mathrm{kg}, 50-\mathrm{cm}$ square by $80-\mathrm{cm}$ tall cuboid, with a gravity-gradient boom for attitude control and solar panels on four sides. 


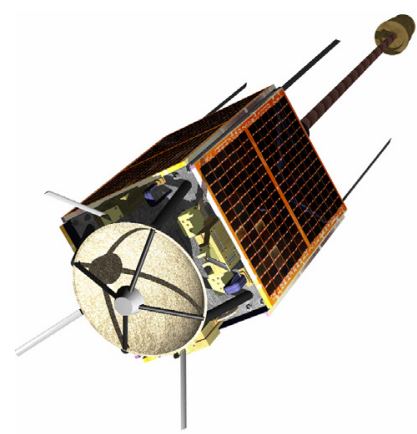

This design looked fine until the various system budgets were calculated. There was not enough power to support the payload, and the antenna was too small to maintain the link required to detect the return signal from the sea surface. It did not have propulsion, and the cost modifications needed to meet even the basic requirements would be prohibitive, if at all possible. The platform design was subsequently discarded for this mission.

A new and creative design was going to be needed to meet the requirements, and this time the electrical power for the mission and the payload antenna size were going to be addressed first.
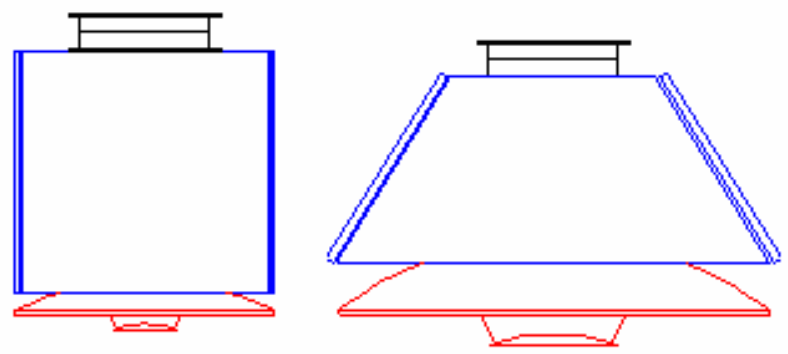

The solar panels were canted out at 30 degrees, which provided more solar cell area, as filling out of the corners increased the panels in size. The new angle increases the power that is generated from the Sun per surface area on top of that, as they are more efficiently pointing at the Sun, keeping the Earth out of their field of view.

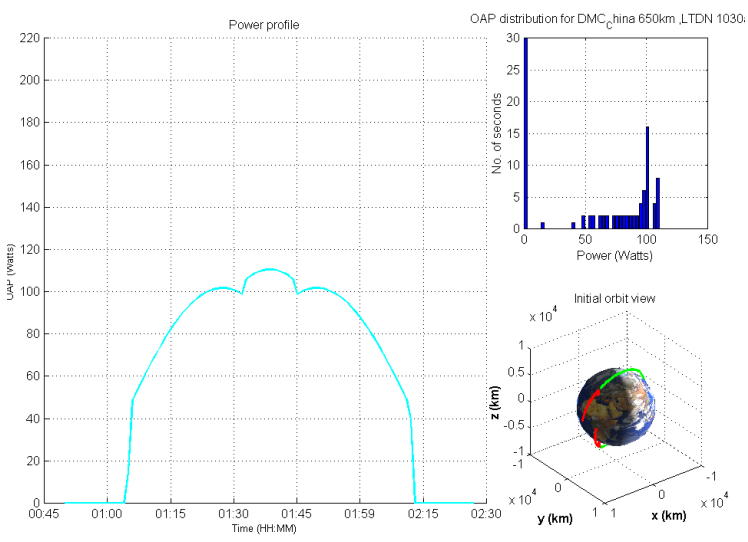

The graph shows the relatively even power generation during the Sunlit portion of one orbit.

This new layout also provides a 1-metre square Nadir facing facet to mount the antenna on, allowing the inclusion of a suitable dish or phased-array antenna.

The internal volume increased significantly: this allows an easy layout of the avionics, payload modules, propulsion and harness, easy access for assembly and test, and simple adjustment options for center-of-gravity adjustments.

\section{Attitude control}

The spacecraft initially had a gravity-gradient boom for attitude control. With the magnetorquers this would provide about 1 degree of pointing accuracy, not enough for this mission, so it was replaced by a pitch momentum wheel. This wheel represents a serious single-point failure mode, so a second wheel is added to the satellite. In order to provide additional redundancy in the solar panels, the second wheel is mounted with its spin axis 90 degrees rotated from the first one. If the first wheel fails, the spacecraft can be rotated 90 degrees, and the mission can proceed as before. If a solar panels fails, the spacecraft can be rotated and run of whichever wheel is required to keep the particular panel out of sunlight, thereby providing full power to the spacecraft again, and without having to include oversize or additional solar panels. As orbits precess, the orientation can be changed every few months as required to keep the mission fully functional

For attitude measurement the satellite has three horizon sensors on board, with their operational axis 120 degrees apart.

\section{Orbit control}

The internal design of the structure allows a simple interface to the propulsion system. As the propulsion is used for orbital corrections only, and not for attitude control, only a single thruster is needed. This thruster can be directed straight up through the attach fitting. If a station keeping maneuver is required, the spacecraft simply performs a 90degree pitch, burns the engine for a while, and returns to normal attitude a few seconds later.

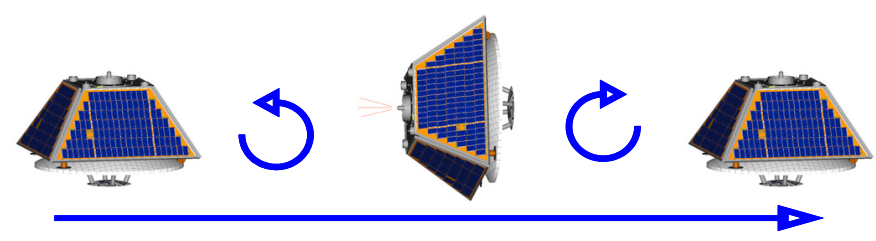

This layout does not only allow the pitch maneuver to be performed by either wheel, but it also keeps the firing direction through the spacecraft's center of gravity as the tank depletes. As this satellite monitors the oceans, it can perform the maneuver over land, without affecting the 
mission. For other payloads appropriate portions of the orbit or operational schedule can usually be found, for instance at the night side of the orbit.

The propulsion system can be integrated at the launch site, allowing the pre-integrated tank, valves and thrusters to be assembled elsewhere, and it can be pre-filled away from the spacecraft. It can be replaced with different systems to provide for different mission requirements at a late stage too, for instance to cope with a late change of launch vehicle, or orbital requirements, or if a secondary launch opportunity into a non-optimal orbit suddenly becomes available.

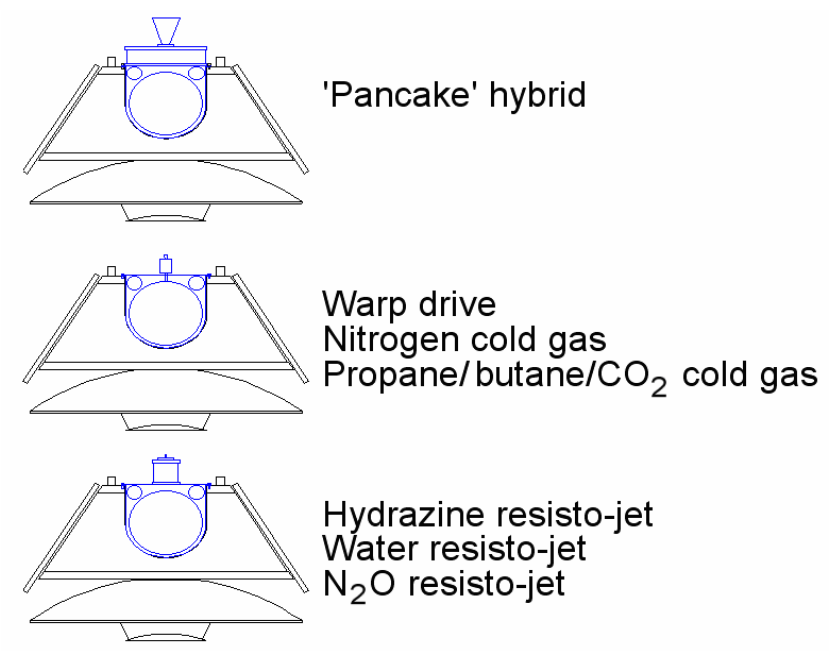

As the diagrams above show, a range of systems can be provided without any change to the spacecraft. The only physical interface consists of a few electrical connectors and a few bolts.

Many constellations do not require individual satellite orbital slot keeping, rather they require that the satellites be kept a certain distance from one another. This means that if the propulsion system of one satellite fails, the rest of the satellites in the constellation can be maneuvered to keep up with the failed one to keep the mission fully operational. The propulsion system is a specific system that by virtue of its size is difficult to duplicate, and having a duplicate system would require both being larger than the original one in order to cope with having to move the additional mass of the second system. It does however have multiple failure modes, as it has moving parts and flowing gasses in it, with changing stresses after launch due to pressure changes.

The internal structure of the satellite consists of two crossed structural aluminum honeycomb sheets. For ease of integration this structure is built up from one main member, and two half members that connect to it.

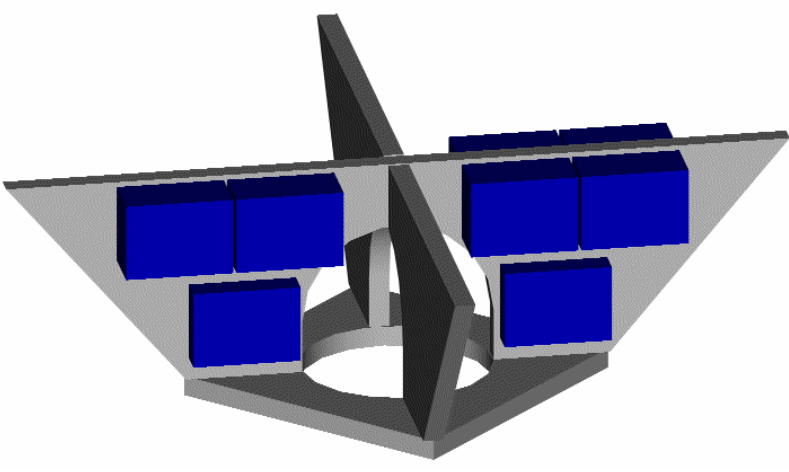

The spacecraft avionics other than orientation-sensitive attitude sensors and actuators are mounted only on the main sheet. The advantage of this mounting is that the satellite can be assembled and partially tested in a flat-sheet mode, with easy access to all units, and taking very little space while in the cleanroom. See the difference floor space required between 1-metre square satellites and the single panels mounted two per stand.

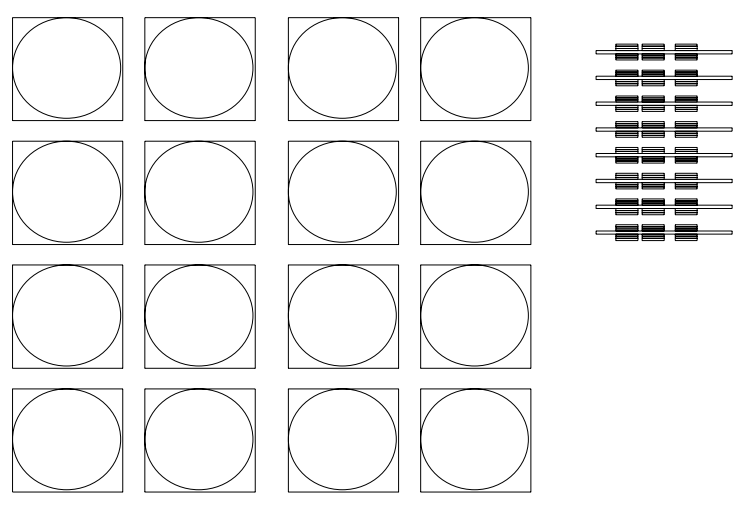

The assembled sheets can be stored in a very small space, which allows a number of satellites to be assembled concurrently in a fairly small facility. It is only at the final stage of assembly that the two cross members are attached and that the payload frame is attached to the top of the frames. The launch vehicle attach frame is mounted to the bottom of the cross-frame, and four solar panels fill out the complete structure. This final assembly could be completed at the launch site, leading to substantially lower shipping costs.

By keeping all payload components on the large top plate, it is possible to assemble those plates separately, possibly at the payload provider's facility. Attitude sensors and actuators are also mounted on this plate, allowing them to be appropriate to the payload rather than to the bus, and any alignment and calibration can be accomplished as an integrated unit. The direct physical connection between the payload and the attitude subsystem makes for an intrinsically stable design that is most likely to withstand launch vibration without introducing errors through shift or rotation. A selection of plates can be held ready for any payloads to be mounted on. The payload plate includes a 
series of standard mounting interfaces. The use of such standard interfaces allows the manufacture of the plates to be independent of the development of payload units, and of platform units such as antennas and sensors.

The individual parts of the structure, including mounted avionics, payloads, and other subsystems, will have been tested for compliance with their design requirements, which means that the final assembly can be done just before launch and not require additional vibration testing. The structural risk of not subjecting the final satellite to a complete vibration test is similar to that of the final assembly of the satellite to the launcher, the assembly of the rocket stages, and the mounting of the fairing. None of these typical launcher assembly tasks are verified by vibration, yet they are acceptable for launch.

\section{FURTHER USE}

The bus design as shown above has been proposed for several missions, and derivatives of it have been launched. They did not use all the quick-modification possibilities, but one of them, TOPSAT, launched just recently in late 2005 , was able to use a different orbit than initially planned for and it perform its mission.

Many other missions have been proposed, showing the versatility of the design.

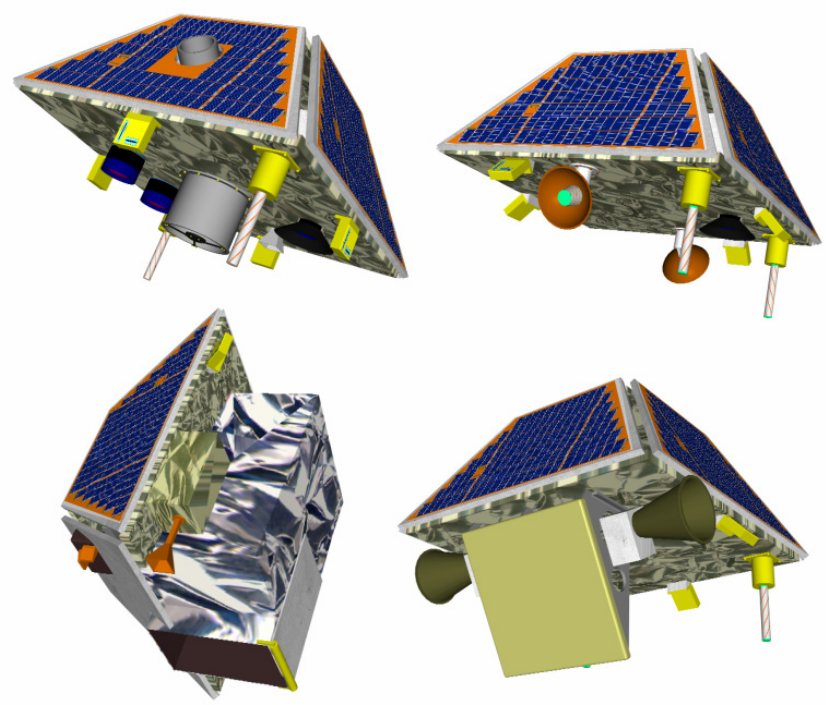

Above are versions for optical Earth observation, atmospheric sounding using GPS signals, and a horizonscanning payload. Below various radar antennas, and ocean color analyzer and a dual hyperspectral imager.

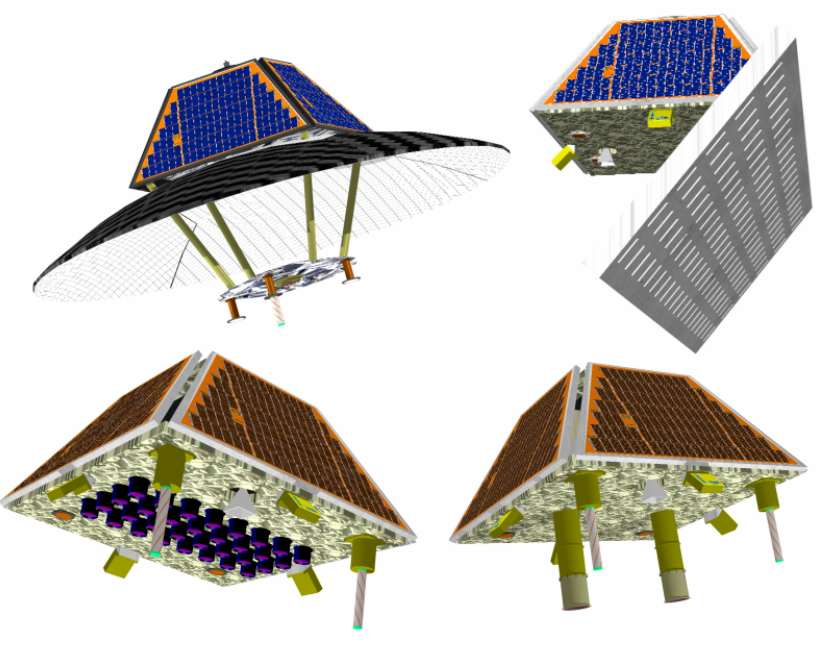

\section{LAUNCH}

The satellite layout allows them to be efficiently packed into a cylindrical volume. This layout allows for instance 8 such spacecraft to be launched on a single Dnepr launcher, as shown in the sketch below. With a selection of payloads, the cost can be shared amongst many customers, and the launcher can be pre-booked without knowing the details about the payloads to be included. With a typical launch capability of three tons to Low Earth Orbit, there is enough mass margin for a strong central tube to carry the eight 100$\mathrm{kg}$ spacecraft, even allowing the spacecraft to grow to 150 or $200 \mathrm{~kg}$ with a heavy payload.

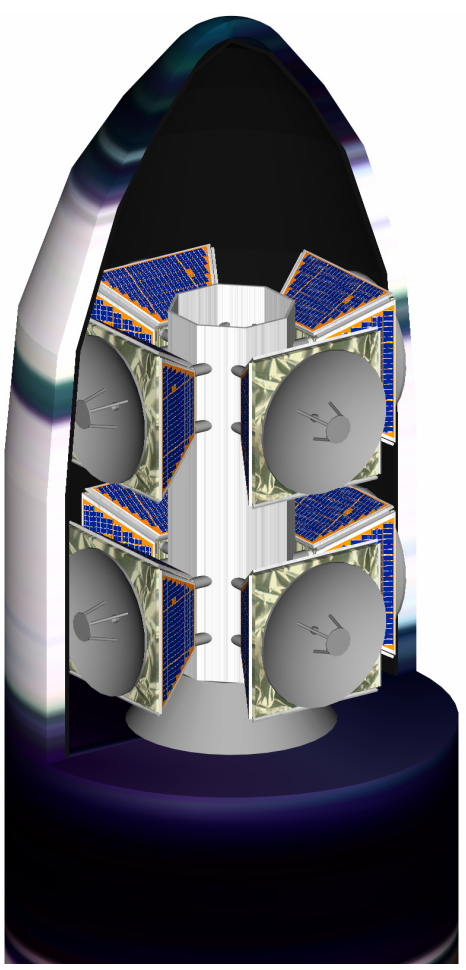


An organization could have a ready stack of satellites mounted on the central dispensing column, with the payload plates not yet assembled. The plates can be selected from a pre-manufactured set, or a late-breaking payload can be quickly added to an existing blank panel for final-moment installation on the satellite body. The whole assembly of central column with assembled spacecraft can then be mounted on the Dnepr, as per usual operation for this vehicle, and launched quickly thereafter. The dual payload floor of the Dnepr can be used to provide two individual sets of spacecraft for different customers.

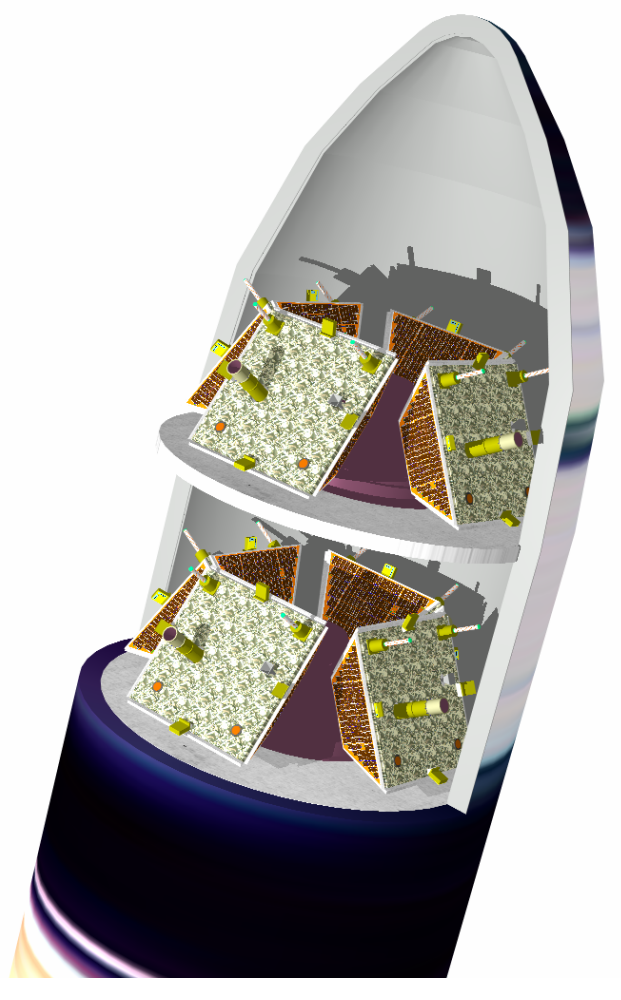

\section{Conclusion}

The Constella platform design developed at SSTL has a number of features that allow it to be pre-manufactured and tested, ready for last-minute customization and quick launch. The design makes for inherent cost savings in both the satellite itself and the required facilities while providing operational redundancy and compatibility with any low Earth orbit. The availability of this platform will enable a very quick response mission that can take advantage of new technology, or to investigate a new cosmic or Earth-bound phenomenon that may be changing or disappearing. Traditional satellites, and even current so called quick turnaround missions that still take a year or more could not contemplate such missions and simply changing procedures to speed things up will not make the fundamental step increases in performance that this design offers.

\section{REFERENCES}

[1] Internal SSTL document on satellite design.

\section{BIOGRAPHY}

Maarten (Max) Meerman is Director of Research at Surrey Satellite Technology Limited and Industrial Reader in Space Systems, both at the University of Surrey,

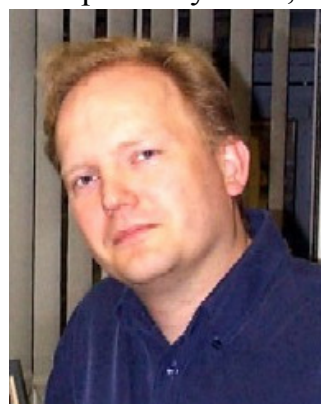
Guildford, UK. He recently returned to Surrey after a two-year break at the U.S. Air Force Academy in Colorado Springs, where he held the Endowed Schriever Chair in Space Systems Engineering at the Astronautics Department, working with cadets in the development of small satellites and sounding rockets. He studied in Amsterdam, the Netherlands, where he received a Bachelor's degree in Electrical Engineering. He is a veteran of numerous space missions, a European Ingenieur, an Associate Fellow of the American Institute of Aeronautics and Astronautics, and a Fellow of the British Interplanetary Society. 